\title{
An In-situ Correction Method of Position Error for an Autonomous Underwater Vehicle Surveying the Sea Floor ${ }^{\dagger}$
}

\author{
Pan-Mook Lee ${ }^{1 *}$, Bong-Huan Jun¹, Jin-Yeong Park ${ }^{1}$, Hyungwon Shim ${ }^{1}$, \\ Jae-Soo Kim², Hun-Sang Jung ${ }^{2}$ and Ji-Young Yoon ${ }^{2}$ \\ ${ }^{1}$ Maritime and Ocean Engineering Research Institute, KORDI, 171 Jang-Dong, Yuseong-Gu, Daejeon 305-343 Korea \\ ${ }^{2}$ Hanwha Corporation R\&D Center, 52-1 Woisam-Dong, Yuseong-Gu, Daejeon 305-156, Korea
}

(Manuscript Received March 14, 2011; Revised April 11, 2011; Accepted May 4, 2011)

\begin{abstract}
This paper presents an in-situ correction method to compensate for the position error of an autonomous underwater vehicle (AUV) near the sea floor. AUVs generally have an inertial navigation system assisted with auxiliary navigational sensors. Since the inertial navigation system shows drift in position without the bottom reflection of a Doppler velocity log, external acoustic positioning systems, such as an ultra short baseline (USBL), are needed to set the position without surfacing the AUV. The main concept of the correction method is as follows: when the AUV arrives near the sea floor, the vehicle moves around horizontally in a circular mode, while the USBL transceiver installed on a surface vessel measures the AUV's position. After acquiring one data set, a least-square curve fitting method is adopted to find the center of the AUV's circular motion, which is transferred to the AUV via an acoustic telemetry modem (ATM). The proposed method is robust for the outlier of USBL, and it is independent of the time delay for the data transfer of the USBL position with the ATM. The proposed method also reduces the intrinsic position error of the USBL, and is applicable to the in-situ calibration as well as the initialization of the AUVs' position. Monte Carlo simulation was conducted to verify the effectiveness of the method.
\end{abstract}

Keywords: Autonomous underwater vehicle (AUV), Position error, In-situ correction, Least square method, Monte Carlo simulation

\section{Introduction}

A navigation system of autonomous underwater vehicles based on inertial sensors is required to initialize the underwater vehicle's position with external information, and is also required to compensate for the accumulation of position errors induced by integrating the inertial measurements which includes uncertain bias errors. The correction of the underwater vehicles' position near the seabed is one of the important issues for precise survey on

\footnotetext{
tThis paper is a revised version of the paper [10] in the proceedings of the Underwater Technology Symposium cancelled due to the earthquake disaster, Tokyo, April 2011.

*Corresponding author. Tel.: +82-42-866-3810, Fax.: +82-42-866-3819.

E-mail address: pmlee@moeri.re.kr.

Copyright @ KSOE 2011.
}

the sea bottom.

Figure 1 depicts the typical process of an AUV used to survey the sea bottom, in which the AUV is launched at a surface ship. After launching at the surface, the AUV can set its initial position using GPS. Dead reckoning or integrated navigation algorithms can be adopted to acquire the underwater position of the vehicle, starting from the initial position. With the aid of an auxiliary Doppler velocity $\log$ (DVL), the AUV may compensate for the drift of position calculated with its inertial sensors or it may formulate a dead reckoning system with a directional gyro.

Because of the current flow and/or tide, however, the AUV only measures relative velocity during the diving process when the DVL cannot acquire the 


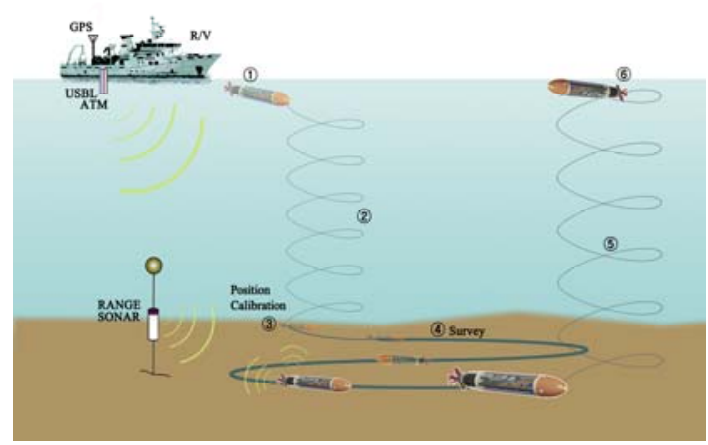

Fig. 1. Operational concept of an AUV for seabed survey.

bottom reflection of its acoustic signal. In the middle of diving process, the inertial navigation system shows drift due to the accumulation of error as time elapses. Therefore, a position error of the vehicle occurs when it arrives at the sea bottom and the error is accumulated while the AUV surveys the sea floor, of which the accumulated error depends on the operation time and the unknown currents.

Using range measurements between an AUV and reference stations, several methods were proposed to reduce the position error. Lee et al. suggested a hybrid navigation system based on a strap-down inertial measurement unit (IMU) accompanied by one or two range measurement sensors as well as a DVL, a depth sensor and a magnetic compass, which can improve the performance of the IMUDVL $[1,2]$. Hegrenæs et al. proposed an underwater transponder positioning that requires only one transponder due to tight coupling with an inertial navigation system [3]. Rui and Chitre presented a cooperative positioning system for multiple AUVs using range-only measurements between two AUVs [4]. By introducing acoustic communication between AUVs, the position and range information was combined by their survey AUV with its own dead reckoning position estimate. Webster et al. reported a theory and implementation of a decentralized navigation system enabling simultaneous single-beacon navigation of multiple underwater vehicles which relied on range measurements from a single beacon to provide an absolute position reference [5].

Ultra Short Base Line (USBL), as one of the acoustic positioning systems (APS), is useful to monitor the AUV's position at the surface ship while the AUV is surveying at the sea floor [6]. If the USBL has an acoustic modem function, we can conduct data communication between the AUV and the surface station as well as monitor the AUV position.

A simple way to achieve this is to set the position of the AUV by the measured position obtained from the USBL. When we directly set the position by an instant signal of the USBL received through acoustic communication, the AUV has an erroneous position due to the time delay of acoustic communication as well as the intrinsic errors of USBL. Flying AUVs such as ISIMI ([7], [8]) always move forward so that the position with USBL includes the error caused by the time difference between the instant of position calculation and the interrogation instant of the AUV's transponder. Furthermore, a time delay for the acoustic data communication is inevitable and the position error caused by the time delay is not negligible especially when the AUV is a considerable distance from the surface ship.

On the other hand, the AUV with ATM must consume high power of the embedded battery for continuous communication. Since the through-put of the acoustic telemetry modem (ATM) is very low and the outlier of USBL is frequent in a harsh ocean environment, a direct position-setting with the USBL measurements obtained through sequential acoustic communication is inefficient and even impractical.

Therefore, for precise positioning of AUVs with USBL and ATM, it is necessary to correct the errors caused by: (1) the intrinsic position error of the USBL measurements that depend on the slant range; $\delta s=R \cdot \delta \theta$, where $\mathrm{R}$ is the slant range, $\delta \theta$ is the error of angular resolution; and (2) the time mismatching between the instant of the USBL measurement and the interrogation instant of the transponder of the AUV, and the integrated time delay between the times measured and received, $\delta t=(R / v)_{U S B L}+(R / v)_{A T M}+\delta t_{U S B L}+\delta t_{A T M}$, where $\mathrm{R}$ is the slant range, $\mathrm{v}$ is the sound velocity, $\delta t_{U S B L}$ is the USBL measurement latency, and $\delta t_{A T M}$ is the latency of the ATM communication. In addition, the correction method should be robust against: (1) the outlier of the USBL measurements; and (2) the time delay due to the failure of data transfer of ATM.

To overcome these shortcomings, this paper has proposed an in-situ correction method for the posi- 
tioning of an AUV near the sea floor, which minimizes the error of the absolute position of the AUV by adopting a least-square curve fitting technique [9]. The main concept of the correction method is as follows. When the AUV surveying the sea floor needs to correct its position, it moves horizontally to make a circular motion, while the USBL of the surface ship acquires the AUV's position. After acquiring one data set, a curve fitting method is adopted to find the center of the AUV's circular motion. The center of the circle is transferred to the AUV via the acoustic telemetry modem. Then, the AUV can calculate its position with the circle center, the radius of circular motion, and its heading. Finally, the AUV can correct the position with the absolute center of rotational motion at the sea floor.

Figure 2 depicts an example of the in-situ calibration conducted at the end of each epoch of a lawn mowing survey of the AUV, without surfacing to calibrate the position with the GPS positioning.

The underwater inertial navigation system can

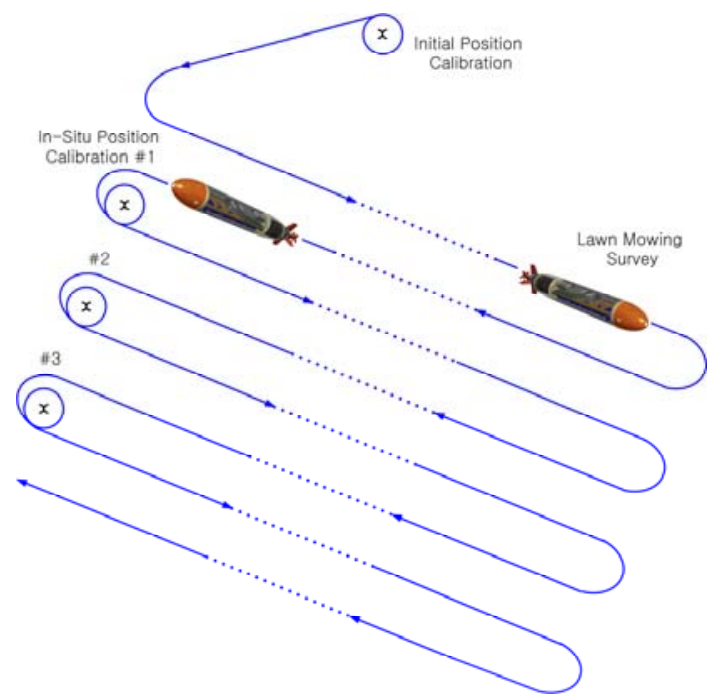

Fig. 2. In-situ position calibration while the AUV surveys the seabed.

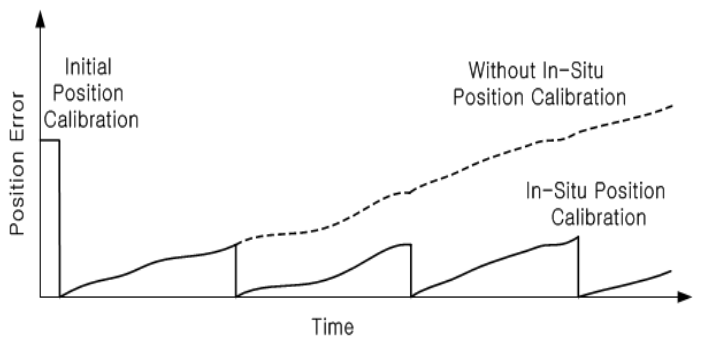

Fig. 3. Effect of the initial and the in-situ position calibrations while the AUV surveys the seabed.

obtain an error-bounded position with the in-situ correction. Figure 3 shows the effect of the proposed correction method. The proposed method can also reduce the intrinsic position error of the USBL. The correction method is robust for the outlier of USBL, and it is independent of the AUV motion that may induce position error due to the time delay of the data transfer with the acoustic modem. The method can be applicable to the in-situ calibration as well as the initialization of the AUVs' position. Monte Carlo simulation was conducted to verify the performance of the correction method [8], [9].

\section{In-situ correction method for AUV's posi- tion error using USBL and ATM}

In this paper, we have assumed that an AUV is equipped with an inertial sensor, a heading sensor, and a DVL embedded in its body, and the AUV can navigate autonomously with its own dead reckoning algorithm. A USBL is intended to be installed at the surface ship, which can communicate data as an ATM between the AUV and the surface ship.

The proposed in-situ correction method involves minimizing the position error of the AUV with a least square curve-fitting algorithm. While the AUV moves with a circular motion after arriving at the sea floor, the USBL of the surface ship acquires the AUV's position. Here we assume that the altitude of the AUV is within the range where the bottom-reflection of the DVL is available. When the surface ship acquires one data set of the circular motion, a circle-fitting method is adopted to the data set to find the center of the AUV's motion. The calculated center is the absolute position of the AUV's circular motion. The center and the radius of the fitted circle are transferred to the AUV via the acoustic telemetry modem. Meanwhile, the AUV recognizes its heading with an internal direc- 
tional sensor so that it can calculate its current position when it identifies the center and the radius of circular motion. Therefore, with the transferred center and radius of circular motion, the AUV can calculate its absolute position.

Figure 4 depicts the communication process between the AUV and the surface ship for the in-situ correction method to minimize the position error. When the AUV arrives at the sea floor, the AUV changes to a calibration mode. In this mode, the AUV begins to make a circular motion in the horizontal plane, and sends its status to the surface ship via the acoustic modem. Upon receiving the status signal, the surface ship replies an acknowledgement signal to the AUV and begins to acquire the AUV position with the USBL. When one set of position data is available, circle-fitting is conducted with the data set to calculate the center and radius of the circle. Also, the surface ship sends the calculated position of the center and radius to the AUV. When the AUV receives the center and the radius, it replies with an acknowledgement signal to the surface ship and begins surveying the sea bed.

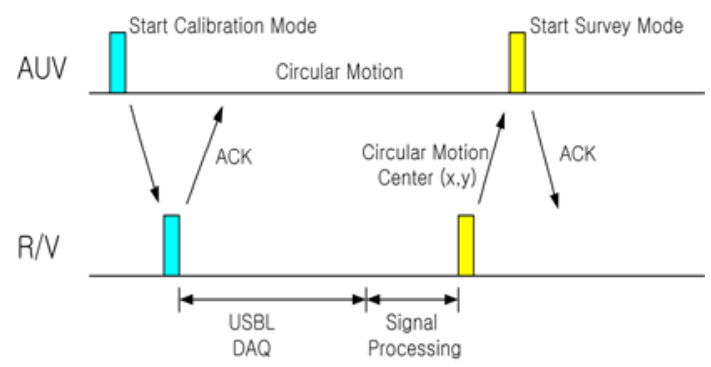

Fig. 4. Data communication process between the AUV and the surface vessel for the in-situ correction of position error.

The proposed in-situ correction method can be performed when the position drift becomes larger than a threshold level while surveying the sea bed with its inertial-acoustic navigation system.

The center of the AUV's circular motion is independent of the AUV's current position, the time delay and mismatch of position measurements of the USBL, and the time delay for data communication of the ATM. Thus, the proposed in-situ correction method is insensitive to the time delay of the USBL measurement, the mismatch of the measurement and the interrogation instants of the USBL, and the time of the acoustic communication with the ATM. We can reject the outliers of the
USBL signals in the circle-fitting process so that the correction method is robust for the outliers. The method is also insensitive to the time delay caused by a temporal failure of acoustic communication with the ATM.

For the implementation of the in-situ correction method, we need a precise directional gyro and DVL sensors. We also need a path tracking controller to make an exact circular motion because a current flow generally exists.

\section{Simulation}

Monte Carlo simulations were carried out to verify the validity of the in-situ correction method to minimize the position error. Three typical survey depths were chosen in simulation: 6,000m depth for survey on the Pacific, 2,000m depth for survey on the East Sea, and 100m depth for survey on a shallow depth area.

We assumed that the forward speed of the AUV is constant with $1.5 \mathrm{~m} / \mathrm{s}$, and the position measurement of USBLs includes random errors with its intrinsic random characteristics. Since a correct outlier simulation is another topic, we excluded the outlier phenomenon in the simulation so that we could compare the positional accuracy of the errors. When the AUV arrives at the sea floor, its position is uncertain because of the ocean current and environmental disturbances. To reduce the complexity in comparison with the simulation results, we fixed the initial offset of the AUV's circle center by $(x, y)=(200,100)$ relative to the surface ship.

We randomly generated the initial position of the AUV around the circumference of the rotating path. The USBL measurements were generated by adding the white random noise in the magnitude of the position error and uniformly distributed noise in phase with the AUV's current position. We repeated the simulation 100 times with a sequential generation of random noises.

Considering the round-trip time of the acoustic signal of the USBL between the surface ship and the AUV, we selected the sampling intervals of 8 seconds, 3 seconds, and 1 second for $6,000 \mathrm{~m}$, $2,000 \mathrm{~m}$, and $100 \mathrm{~m}$ depths, respectively. For the deep-sea survey, we assumed a USBL was applied with the high accuracy of a $0.3 \%$ slant range [10]. Also, for the shallow water survey, we assumed a 
USBL was applied with the low accuracy of a $2.5 \%$ slant range [11].

\subsection{Position correction for deep sea survey}

Monte Carlo simulation was performed for a deep sea survey condition with the accurate USBL. The effect of various conditions over the radius of the circle and the effect of the total sample time were examined to evaluate the correction characteristics for the position error. The simulation conditions for a deep-sea survey at a $6,000 \mathrm{~m}$ depth are as follows:

1) Simulation condition: AUV speed $1.5 \mathrm{~m} / \mathrm{s}$, depth $6,000 \mathrm{~m}$, standard deviation $(\sigma)$ of USBL $=18 \mathrm{~m}$, update period of USBL measurement: 8 seconds

2) Variables: radius of circular motion $R(=25,50$, $100,150 \mathrm{~m})$, total sample time $\mathrm{T}(=300,600$, 1200, 1800s)

The simulated measurements were obtained by adding random noises to the AUV's temporal position. The magnitude of the position error for the $6,000 \mathrm{~m}$ depth survey with the accurate USBL was set with $18 \mathrm{~m}$ in standard deviation. Figure 5(a) shows the curve fitting results for a $100 \mathrm{~m}$ radius of the motion at a $6,000 \mathrm{~m}$ depth, where the sample time for the fitting was 600 seconds. In this figure, the solid line with the ' $*$ ' marks was the trajectory of the measured AUV's position, and the red dotted line was the true path of the AUV. The black solid circle was the fitted circle for the measurement data.

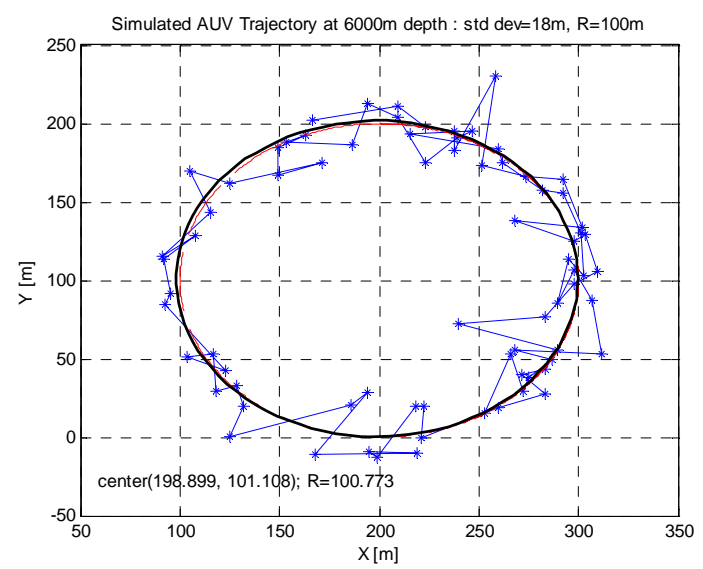

(a)
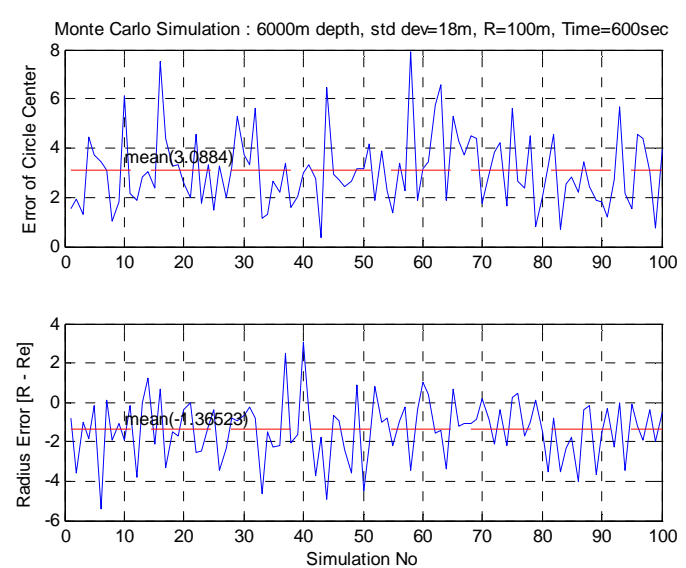

(b)

Fig. 5. Circle fitting result with $\mathrm{R}=50 \mathrm{~m}$; (a) $\mathrm{x}-\mathrm{y}$ plane trajectory and fitted circle, (b) estimation errors of the center and radius of circle in Monte Carlo simulation.

In case of Figure 5(a), the estimated center and radius obtained from the curve fitting was (198.899m, 101.108m) and $100.773 \mathrm{~m}$, respectively. The estimation error of the circle center was ($1.101 \mathrm{~m}, 1.108 \mathrm{~m}$ ), of which the circular accuracy is about $1.562 \mathrm{~m}$. This means that we can initialize the AUV position within $1.6 \mathrm{~m}$ accuracy when the AUV is at the sea floor. The estimation error of the radius, however, was $0.773 \mathrm{~m}$. This phenomenon was caused by squaring the error in the curve fitting with the least mean square optimization. The estimated radius is generally larger than the true radius of the circular motion. Therefore, to obtain a more precise positioning for the AUV with the in-situ correction method, we should use the radius of the AUV's circular path rather than the estimated radius.

Figure 5(b) shows the errors of the estimated center and the estimated radius obtained from the Monte Carlo simulation by a 100 times run. The minimum and maximum errors of the estimated center were $0.3 \mathrm{~m}$ and $8.0 \mathrm{~m}$, respectively, and the maximum error of the estimated radius was $5.4 \mathrm{~m}$. The mean error of the center and the radius were $3.088 \mathrm{~m}$ and $1.365 \mathrm{~m}$, respectively. Therefore, we can initialize the AUV position about 3m accuracy on average with the accurate USBL when the AUV makes a circular motion with a $100 \mathrm{~m}$ radius for 10 minutes at a $6,000 \mathrm{~m}$ depth.

Table 1 depicts the mean values of the estimated center errors for the Monte Carlo simulation results with the various conditions described in 1) and 2), 
where the AUV was at a 6,000m depth. Figure 6 is a 3-dimensional plot of the results shown in Table 1. We found that the estimation error of the center monotonically decreases as the sample time increases, and it generally decreases as the radius of

Table 1. Estimation errors of circle center in Monte Carlo simulation: $6,000 \mathrm{~m}$ depth, $18 \mathrm{~m} \sigma$ of USBL error.

\begin{tabular}{|c|c|c|c|c|}
\hline Radius Time & $300 \mathrm{sec}$ & $600 \mathrm{sec}$ & $1200 \mathrm{sec}$ & $1800 \mathrm{sec}$ \\
\hline $25 \mathrm{~m}$ & 6.233 & 3.996 & 2.871 & 2.304 \\
\hline $50 \mathrm{~m}$ & 4.892 & 2.985 & 2.219 & 1.856 \\
\hline $100 \mathrm{~m}$ & 4.920 & 3.088 & 1.891 & 1.528 \\
\hline $150 \mathrm{~m}$ & 9.575 & 2.810 & 1.888 & 1.554 \\
\hline
\end{tabular}

the circular motion increases. The one exception is the condition having a $150 \mathrm{~m}$ radius with a 300 seconds sample time, where the data set was insufficient for curve fitting to match at least one cycle of circular motion. Table 2 depicts the estimation errors of the radius of the AUV's circular motion. The estimated radius is always larger than the true one except the incomplete curve fitting.

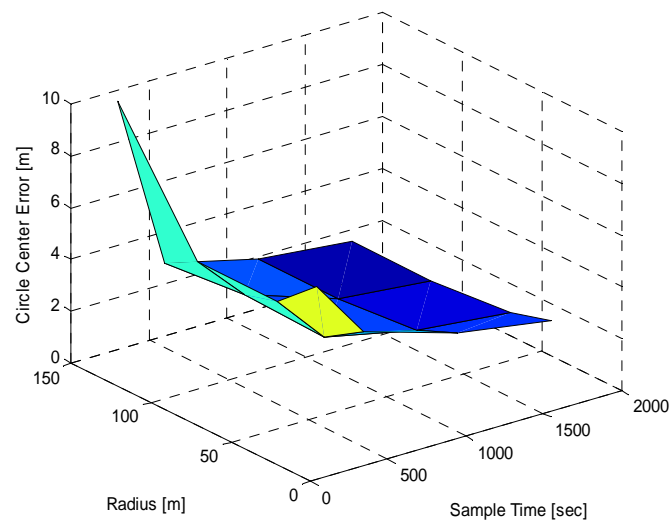

Fig. 6. Estimation errors of circle center in Monte Carlo simulation: 6,000m depth, $18 \mathrm{~m}$ of USBL error.

Table 2. Estimation errors of circle radius in Monte Carlo simulation: $6,000 \mathrm{~m}$ depth, $18 \mathrm{~m} \sigma$ of USBL error.

\begin{tabular}{|c|c|c|c|c|}
\hline Radius Time & $300 \mathrm{sec}$ & $600 \mathrm{sec}$ & $1200 \mathrm{sec}$ & $1800 \mathrm{sec}$ \\
\hline $25 \mathrm{~m}$ & -6.325 & -6.128 & -5.869 & -5.845 \\
\hline $50 \mathrm{~m}$ & -3.518 & -3.369 & -3.187 & -3.163 \\
\hline $100 \mathrm{~m}$ & -0.981 & -1.365 & -1.458 & -1.398 \\
\hline $150 \mathrm{~m}$ & 3.895 & -0.909 & -0.949 & -0.976 \\
\hline
\end{tabular}

In terms of the AUV surveying sea bed at $6,000 \mathrm{~m}$ with the accurate USBL, from the simulation results, it was induced that we can correct the position within $2 \mathrm{~m}$ accuracy when the AUV makes a circular motion at least for 20 minutes with a radius larger than $100 \mathrm{~m}$. To obtain a $5 \mathrm{~m}$ accuracy of the position, the AUV should make a circular motion with a radius larger than $50 \mathrm{~m}$ and a sample time longer than at least one cycle of circular motion.

We conducted another simulation on the in-situ correction for the case of the 2,000m depth survey with the accurate USBL. The simulation conditions for a deep-sea survey at a $2,000 \mathrm{~m}$ depth are as follows:

3) Simulation condition: AUV speed $1.5 \mathrm{~m} / \mathrm{s}$, depth $2,000 \mathrm{~m}$, standard deviation $(\sigma)$ of USBL $=6 \mathrm{~m}$, update period of USBL measurement: 3 seconds

4) Variables: radius of circular motion $R(=15,25$, $35,50 \mathrm{~m})$, total sample time $\mathrm{T}(=100,200,300$, 600s)

Table 3 depicts the mean values of the estimated center errors for the Monte Carlo simulation results with the various conditions described in 3) and 4), where the AUV was at a 2,000m depth. Figure 7 is a 3-dimensional plot of the results shown in Table 3 . In terms of the AUV surveying sea bed at $2,000 \mathrm{~m}$ with the accurate USBL, we can correct the position within $2 \mathrm{~m}$ accuracy whenever the AUV makes a circular motion having a sample time longer than at least one cycle of circular motion. To obtain $1 \mathrm{~m}$ accuracy of the position at a 2,000m depth, we should conduct the in-situ correction for the AUV with a sample time longer than 300 seconds.

Table 3. Estimation errors of circle center in Monte Carlo simulation: $2,000 \mathrm{~m}$ depth, $6 \mathrm{~m} \sigma$ of USBL error.

\begin{tabular}{|c|c|c|c|c|}
\hline Radius $\quad$ Time & $100 \mathrm{sec}$ & 200sec & $300 \mathrm{sec}$ & $600 \mathrm{sec}$ \\
\hline $15 \mathrm{~m}$ & 1.792 & 1.246 & 0.962 & 0.689 \\
\hline $25 \mathrm{~m}$ & 1.596 & 1.132 & 0.902 & 0.589 \\
\hline $35 \mathrm{~m}$ & 1.830 & 1.125 & 0.778 & 0.552 \\
\hline $50 \mathrm{~m}$ & 3.363 & 0.971 & 0.861 & 0.547 \\
\hline
\end{tabular}




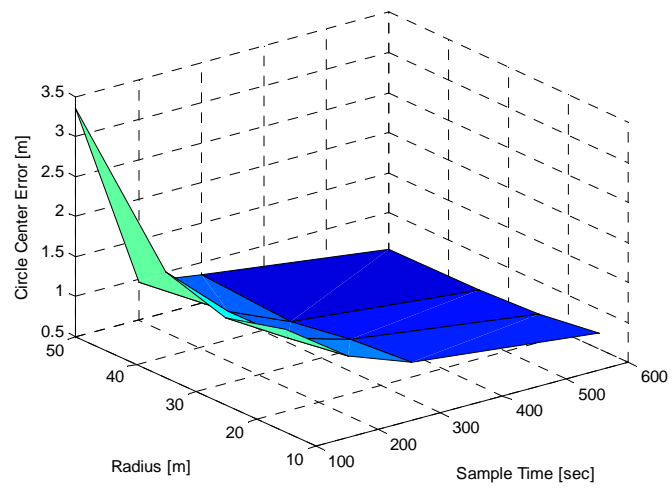

Fig. 7. Estimation errors of circle center in Monte Carlo simulation: 2,000m depth, $6 \mathrm{~m} \sigma_{\mathrm{of}} \mathrm{USBL}$ error.

\subsection{Position correction for shallow water survey}

Similarly to the previous studies, Monte Carlo simulation was performed for a shallow water survey condition with a low accuracy USBL. The simulation conditions for a shallow water survey at 100m depth are as follows:

5) Simulation condition: AUV speed $1.5 \mathrm{~m} / \mathrm{s}$, depth $100 \mathrm{~m}$, standard deviation $(\sigma)$ of USBL $=2.5 \mathrm{~m}$, update period of USBL measurement: 1 seconds

6) Variables: radius of circular motion $\mathrm{R}(=15,25$, $35,50 \mathrm{~m})$, total sample time $\mathrm{T}(=60,120,180$, 240s)

Figure 8 shows the errors of the estimated center and the estimated radius having a $5 \mathrm{~m}$ radius for 60 seconds at a $100 \mathrm{~m}$ depth, which were obtained from the Monte Carlo simulation by a 100 times run. The results show that the maximum radius error for the position was less than $1 \mathrm{~m}$, and the corrected error of radius was $0.388 \mathrm{~m}$ on average.

Table 4 depicts the mean values of the estimated center errors for the Monte Carlo simulation results with the various conditions described in 5) and 6), where the AUV was at a $100 \mathrm{~m}$ depth. From this table, we can correct the position within $1 \mathrm{~m}$ accuracy for every simulation. In terms of the AUV surveying the sea bed at $100 \mathrm{~m}$ depth, the simulation results demonstrate that we can correct the position within $0.4 \mathrm{~m}$ accuracy when the AUV makes a circular motion at least for 2 minutes with a radius larger than $10 \mathrm{~m}$.

\section{Conclusions}

This paper has proposed an in-situ correction method to reduce the position error of an AUV near sea floor, where the AUV makes a circular motion and the measured position data from the USBL were fitted to a circle. We also carried out Monte Carlo simulation to evaluate the validity of the proposed method. When the AUV surveyed the sea bed at $2,000 \mathrm{~m}$ with a USBL having the accuracy with a $0.3 \%$ slant range in standard deviation, we can correct the position within $2 \mathrm{~m}$ accuracy whenever the AUV makes a circular motion having a sample time longer than at least one cycle of circular motion.

When we conduct the in-situ correction for the AUV with a sample time longer than 300 seconds, we can obtain $1 \mathrm{~m}$ accuracy of the position at a 2,000m depth. Meanwhile, when the AUV surveys the sea bed at $6,000 \mathrm{~m}$ with the same USBL, we can correct the position within $2 \mathrm{~m}$ accuracy whenever the AUV makes a circular motion having the sample time longer than at least one cycle of circular motion. When the AUV makes a circular motion with a radius larger than $50 \mathrm{~m}$ and a sample time longer than at least one cycle of circular motion, then we can obtain $5 \mathrm{~m}$ accuracy of the position at a $6,000 \mathrm{~m}$ depth. For the AUV near the sea bed at $100 \mathrm{~m}$ with a USBL having accuracy with a $2.5 \%$ slant range in standard deviation, we can correct the position within $0.4 \mathrm{~m}$ accuracy on average when the AUV is in correction mode at least for 2 minutes with a radius larger than $10 \mathrm{~m}$. The in-situ correction method is robust for the outliers of the USBL measurements, and it is also insensitive to the time delay of USBL and ATM and the time delay caused by a temporal failure of acoustic communication with ATM. 

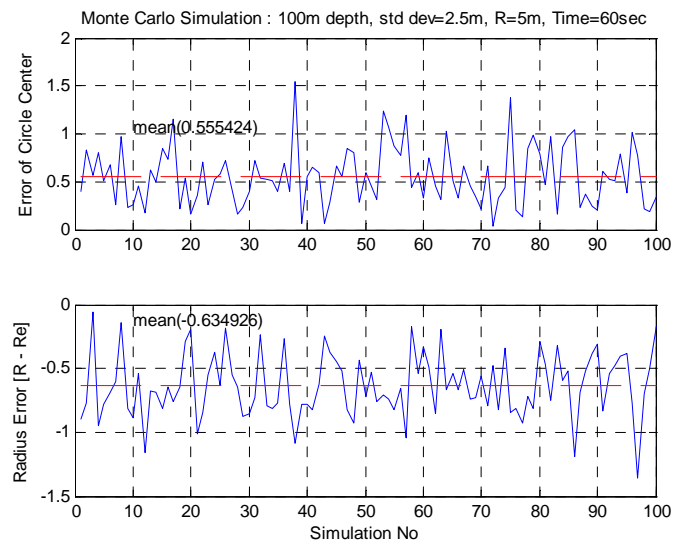

Fig. 8. Estimation errors of the center and radius of circle in Monte Carlo simulation: $100 \mathrm{~m}$ depth, $2.5 \mathrm{~m} \sigma$ of USBL error, $5 \mathrm{~m}$ radius, 1 minutes sampling time.

Table 4. Estimation errors of circle center in Monte Carlo simulation: $100 \mathrm{~m}$ depth, $2.5 \mathrm{~m} \sigma$ of USBL error.

\begin{tabular}{|c|c|c|c|c|}
\hline Radius & $60 \mathrm{sec}$ & $120 \mathrm{sec}$ & $180 \mathrm{sec}$ & $240 \mathrm{sec}$ \\
\hline $5 \mathrm{~m}$ & 0.555 & 0.388 & 0.333 & 0.280 \\
\hline $10 \mathrm{~m}$ & 0.492 & 0.282 & 0.254 & 0.226 \\
\hline $15 \mathrm{~m}$ & 0.428 & 0.304 & 0.250 & 0.213 \\
\hline $20 \mathrm{~m}$ & 0.465 & 0.300 & 0.225 & 0.189 \\
\hline
\end{tabular}

\section{Acknowledgements}

This work was supported by the Ministry of Land, Transportation and Maritime Affairs (MLTM) of Korea for the development of an advanced deep-sea unmanned underwater vehicle, and partially supported by KORDI for surmounting technology over maritime extreme environment.

\section{References}

[1] P. M. Lee, B. H. Jun, K. Kim, C. M.. Lee, T. Aoki, and T. Hyakudome, Simulation of an inertial acoustic navigation system with range aiding for an autonomous underwater vehicle, IEEE J. of Oceanic Engineering, 32(2) (2007) 392-345.

[2] P. M. Lee and B. H. Jun, Pseudo long base line navigation algorithm for underwater vehicles with inertial sensors and two acoustic range measurements, Ocean Engineering, 34(3-4) (2007), 416-425.
[3] Ø. Hegrenæs, K. Gade, O. K. Hagen, and P. E. Hagen, Underwater Transponder Positioning and Navigation of Autonomous Underwater Vehicles, Proc. of IEEE/MTS Oceans Conference, Biloxi, Oct. (2009).

[4] G. Rui and M. Chitre, Cooperative Positioning Using Range-Only Measurement between Two AUVs, Proc. of IEEE Oceans, Sydney, May (2010).

[5] S. E. Webster, L. L. Whitcomb, and R. M. Eustice, Advances in Decentralized Single-Beacon Acoustic Navigation for Underwater Vehicles Theory and Simulation, Proc. IEEE/OES AUV 2010 Conf., Monterey, CA, Aug. (2010).

[6] P. Milne, Underwater acoustic positioning systems. Gulf Publishing Co., Huston, (1983).

[7] B. H. Jun, J. Y. Park, P. M. Lee, F. Y. Lee, and J. H. Oh, Development and tank test of an autonomous underwater vehicle ISIMI, J. of Ocean Engineering, 21(2), (2007), 67-74 (Korean).

[8] P. M. Lee, et al., Development of an advanced deep-sea unmanned underwater vehicle (II-3), Technical Report of KORDI, UCPM S184A2197-2, April (2010) (Korean).

[9] P. M. Lee, B. H. Jun, J. Y. Park, J. S. Kim, H. S. Jung, and Y. Y. Yoon, A Study on the calibration of initial position of an autonomous underwater vehicle at sea floor, Proceedings of 2010 Conference of the Korea Society of Ocean Engineering, Nov. (2010), 94-98; Journal of Ships and Ocean Engineering, KORDIMOERI, June (2011), (To-be published) (Korean).

[10]P. M. Lee, B. H. Jun, J. Y. Park, H. Shim, J. S. Kim, H. S. Jung, and Y. Y. Yoon, An in-situ correction method of initial position error for an autonomous underwater vehicle near sea floor, Proceedings of the International Symposium on Underwater Technology 2011, April 58 , (2011) [The symposium was cancelled due to the earthquake disaster, but proceedings has been published].

[11]IXSEA, USBL Posidonia 6000 Positioning System, User's Manual, (2004).

[12]Applied Acoustics Engineering Ltd., Easytrak USBL,(2010); http://www.appliedacoustics.com/Products_Eas y.aspx?content=portable 